\title{
INVOLUTIONS OF KNOTS THAT FIX UNKNOTTING TUNNELS
}

\author{
DAVID FUTER
}

\begin{abstract}
Let $K$ be a knot that has an unknotting tunnel $\tau$. We prove that $K$ admits a strong involution that fixes $\tau$ pointwise if and only if $K$ is a two-bridge knot and $\tau$ its upper or lower tunnel.
\end{abstract}

\section{INTRODUCTION}

Let $L$ be a link of one or two components in $S^{3}$. An unknotting tunnel for $L$ is a properly embedded arc $\tau$, with $L \cap \tau=\partial \tau$, such that the complement of a regular neighborhood of $L \cup \tau$ is a genus 2 handlebody. As described in [2], an unknotting tunnel induces a strong inversion of the link complement - that is, an involution of $S^{3}$ that sends each component of $L$ to itself with reversed orientation.

When $L$ is a two-component link, it is known that this involution can be chosen to fix $\tau$ pointwise. As a result, an argument of Adams in [1] gives us some geometric information about the tunnel: when the complement of $L$ is hyperbolic, $\tau$ is isotopic to a geodesic in the geometric structure. [1 conjectures that the same is true for knots: that any unknotting tunnel for a hyperbolic knot is isotopic to a geodesic. As for links, this conjecture would follow easily if it were known that $\tau$ is fixed pointwise by some strong inversion of the knot.

The main result of this paper is that this happens only in the wellknown special case of 2-bridge knots. (See Section 3 for background on on 2-bridge knots and their unknotting tunnels.)

Theorem 1.1. Let $K \subset S^{3}$ be a knot with an unknotting tunnel $\tau$. Then $\tau$ is fixed pointwise by a strong inversion of $K$ if and only if $K$ is a two-bridge knot and $\tau$ is its upper or lower tunnel.

This theorem materialized amid the wonderful hospitality of the Isaac Newton Institute for Mathematical Sciences at Cambridge University. I have benefited greatly from conversations with Ian Agol, Yoav Moriah, and Makoto Sakuma. Steve Kerckhoff and Saul Schleimer both

Date: November 8, 2018. 
devoted a great deal of time to hearing and vetting many versions of the proof-in-progress, and deserve my sincerest gratitude.

\section{Tunnels And Involutions}

Notation. From now on, $K$ will denote a knot in $S^{3}$ that has an unknotting tunnel $\tau$. Thus $K \cup \tau$ is realized as a graph with two vertices and three edges $\tau, K_{1}$, and $K_{2}$, where $K=K_{1} \cup K_{2}$. When identifying $\tau$ as a particular tunnel $\tau_{0}$ of the knot $K$, we mean that $K \cup \tau$ is equivalent to $K \cup \tau_{0}$ via an isotopy of $S^{3}$ that preserves $K$ setwise. Note that this notion of equivalence is stronger than isotopy of tunnels in the knot exterior, because the endpoints of $\tau$ are not allowed to pass through each other.

Definition. For any graph $\Gamma \subset S^{3}$, let $N(\Gamma)$ be an open regular neighborhood of $\Gamma$. We call $E(\Gamma)=S^{3} \backslash N(\Gamma)$ the exterior of $\Gamma$.

An unknotting tunnel induces a genus 2 Heegaard splitting of $S^{3}$ into handlebodies $V_{1}=\overline{N(K \cup \tau)}$ and $V_{2}=E(K \cup \tau)$. Let $\Sigma$ be the Heegaard surface: $\Sigma=\partial N(K \cup \tau)$. A genus 2 handlebody admits a hyper-elliptic involution that preserves the isotopy class of every simple closed curve on its boundary; it is unique up to isotopy. Thus the hyperelliptic involutions of $V_{1}$ and $V_{2}$ can be joined over $\Sigma$ to an orientationpreserving involution $\varphi_{\tau}$ on all of $S^{3}$. This involution sends $K$ to itself with reversed orientation; i.e. is a strong inversion of $K$ (see [2]).

The action of $\varphi_{\tau}$ preserves the meridians of $K_{1}$ and $K_{2}$ on $\Sigma$ while reversing the orientation on $K$ - so it must switch the two vertices of $K \cup \tau$ and thus reverse the orientation on $\tau$. It is conceivable, however, that some other involution $\psi$ might fix $\tau$ pointwise while switching $K_{1}$ with $K_{2}$. Theorem [1.1 says that this only happens for 2-bridge knots.

\section{Two-Bridge Knots}

Definition. A rational tangle is a pair $(B, t)$, where $B$ is a 3 -ball and $t$ consists of two disjoint, properly embedded arcs $\gamma_{1}$ and $\gamma_{2}$. We further require that the $\gamma_{i}$ are both isotopic to $\partial B$ via disjoint disks $D_{i}$.

Definition. A knot $K \subset S^{3}$ is called a two-bridge knot if some sphere $S$ splits $\left(S^{3}, K\right)$ into two rational tangles. $S$ is then called a bridge sphere for $K$.

A 2-bridge knot has a 4-plat projection with two maxima at the top and two minima at the bottom [3]. Any horizontal plane, together with the point at infinity, then serves as a bridge sphere for $K$. The 4-plat projection also reveals two unknotting tunnels for $K$ : an upper tunnel 


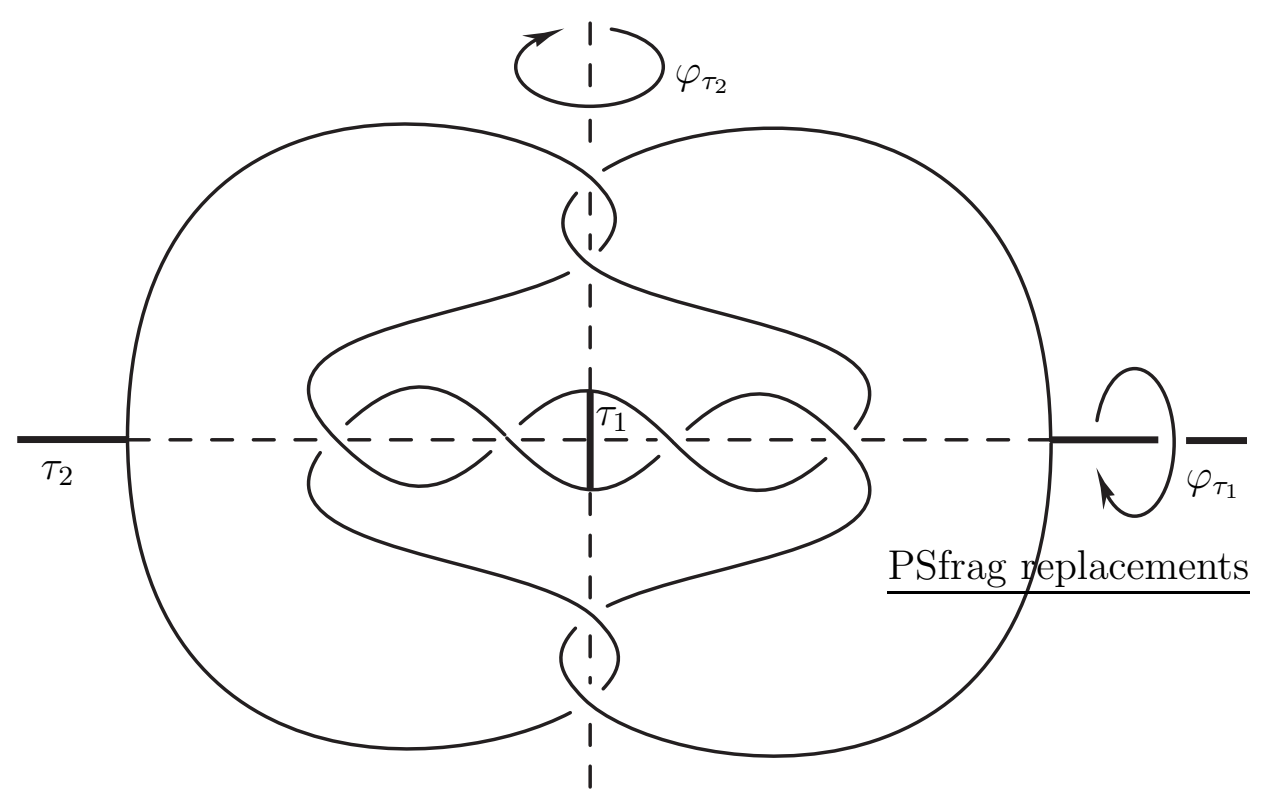

FiguRE 1. Upper and lower tunnels, and the corresponding involutions, in the tri-symmetric projection.

$\tau_{1}$ connecting the two maxima, and a lower tunnel $\tau_{2}$ connecting the two minima.

Two-bridge knots also have four dual tunnels, which may be isotopic to the upper or lower tunnels in special cases. Morimoto and Sakuma classified the six total tunnels up to homeomorphism and isotopy of the knot exterior [7. Kobayashi showed that any unknotting tunnel of a 2-bridge knot is exterior-isotopic to one of these six 4 .

The following result is an immediate consequence of [2, Section 3].

Lemma 3.1. Let $K$ be a two-bridge knot, and $\tau_{1}$ and $\tau_{2}$ be its upper and lower tunnels. Let $\varphi_{\tau_{i}}$ the involution of $S^{3}$ that comes from the handlebody decomposition induced by $\tau_{i}$. Then $\varphi_{\tau_{1}}$ can be chosen to fix $\tau_{2}$ pointwise, and $\varphi_{\tau_{2}}$ can be chosen to fix $\tau_{1}$ pointwise.

Proof. As described in [3, there is an isotopy of $K$ from the 4-plat projection to a tri-symmetric projection, carrying $\tau_{1}$ to part of the vertical axis of symmetry and $\tau_{2}$ to part of the horizontal axis. (See Figure 1.) Abusing notation slightly, we continue to call these isotoped tunnels $\tau_{1}$ and $\tau_{2}$. Now, the involution $\varphi_{\tau_{1}}$ is evident in the figure as a $180^{\circ}$ rotation about the horizontal axis containing $\tau_{2}$, and thus fixes $\tau_{2}$ pointwise. Similarly, $\varphi_{\tau_{2}}$ is a rotation about the vertical axis containing $\tau_{1}$, fixing $\tau_{1}$ pointwise. 


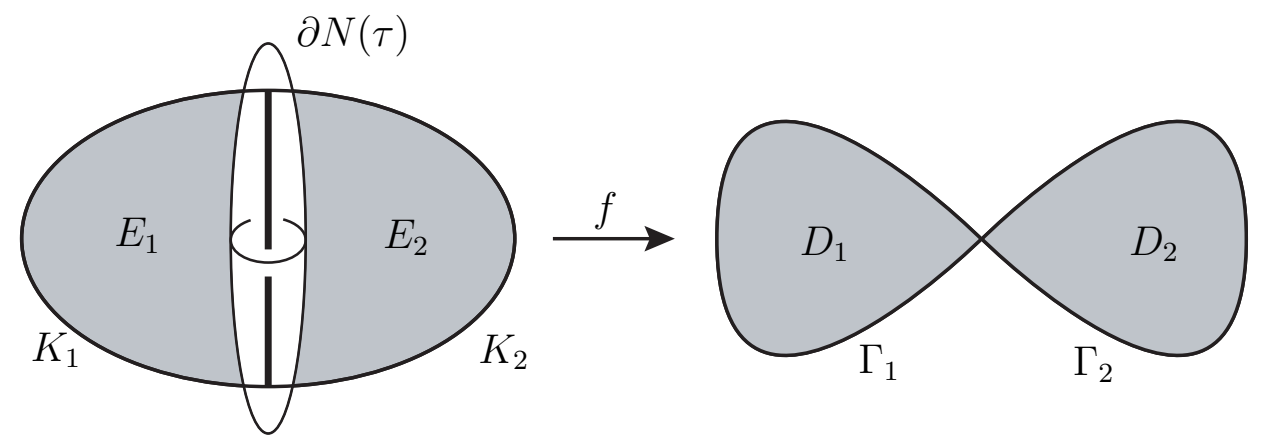

Figure 2. The graphs and disks of Corollary 3.3 .

For the purpose of recognizing two-bridge knots based on involutions, our main tool is a theorem of Scharlemann and Thompson about planar graphs.

Definition. A finite graph $\Gamma \subset S^{3}$ is called planar if it lies on an embedded 2-sphere in $S^{3}$. $\Gamma$ is called abstractly planar if it is homeomorphic to a planar graph.

Theorem 3.2. 8] A finite graph $\Gamma \subset S^{3}$ is planar if and only if

(1) $\Gamma$ is abstractly planar,

(2) every proper subgraph of $\Gamma$ is planar, and

(3) $E(\Gamma)$ is a handlebody.

Remark. For our purposes, we will only need the special case of this theorem when $\Gamma$ consists of one vertex and two edges. This was first proved in an unpublished preprint of Hempel and Roeling.

Corollary 3.3. Let $K$ be a knot with unknotting tunnel $\tau$, where $\tau$ splits $K$ into edges $K_{1}$ and $K_{2}$. If both $K_{1} \cup \tau$ and $K_{2} \cup \tau$ are unknots, then $K$ is a two-bridge knot with splitting sphere $\partial N(\tau)$. Furthermore, $\tau$ is an upper or lower tunnel.

Proof. Let $f: S^{3} \rightarrow S^{3}$ be a map that contracts $\overline{N(\tau)}$ to a point and is the identity outside a small regular neighborhood of $\overline{N(\tau)}$. Then $\Gamma=f(K \cup \tau)$ is a graph with one vertex and two edges, which is clearly abstractly planar. Each proper subgraph $\Gamma_{i} \subset \Gamma$ is the image of $K_{i} \cup \tau$, and is thus an unknotted circle. Also, $E(\Gamma)$ is a handlebody since $E(K \cup \tau)$ is a handlebody. Thus, by Theorem 3.2 . $\Gamma$ is planar.

Since $\Gamma$ is planar, its subgraphs $\Gamma_{1}$ and $\Gamma_{2}$ bound disjoint disks $D_{1}$ and $D_{2}$ in $S^{3}$. (See Figure 2, These disks pull back via $f$ to disjoint disks $E_{1}, E_{2} \subset E(\tau)$. Each $E_{i}$ thus provides an isotopy of $K_{i} \cap E(\tau)$ to $\partial N(\tau)$, making $(E(\tau), K \cap E(\tau))$ a rational tangle. Meanwhile, $N(\tau)$ 
intersects $K$ in two short arcs that are clearly boundary-parallel, so that is a rational tangle too. Therefore, $K$ is a 2-bridge knot with splitting sphere $\partial N(\tau)$.

It is known (from [9], for example) that a splitting of a 2-bridge knot into rational tangles is unique up to isotopy. Thus an ambient isotopy of $S^{3}$ carries $K$ to a 4-plat projection and $N(\tau)$ to a half-space containing just the two maxima (or minima). Thus $\tau$ is an upper or lower tunnel for $K$.

\section{Cyclic Groups Acting on Handlebodies}

A key ingredient in the proof of Theorem 1.1 is the equivariant loop theorem of Meeks and Yau.

Theorem 4.1. 5] Let $M$ be a compact, connected 3-manifold with connected boundary, and $G$ be a finite group acting smoothly on $M$. Let $H$ be the kernel of the homomorphism of fundamental groups induced by the inclusion $\partial M \hookrightarrow M$. Then there is a collection $\Delta=\left\{D_{1}, \ldots, D_{n}\right\}$ of disjoint, properly embedded, essential disks in $M$ such that

(1) $\partial D_{i} \subset \partial M$ for all $i$,

(2) $H$ is the normal subgroup of $\pi_{1}(M)$ generated by the $\partial D_{i}$, and

(3) $\Delta$ is invariant under the action of $G$.

Notation. Let $M$ and $\Delta$ be as above, and denote by $S$ be the union of the disks in $\Delta$. Let $M \mid \Delta=M \backslash N(S)$, where the regular neighborhood is chosen to be invariant under the action of $G$.

To adapt this theorem for our purposes, we assume that $M$ is a handlebody and analyze the pieces of $M \mid \Delta$.

Lemma 4.2. Let $V$ be a handlebody, and let $G$ be a finite group acting smoothly on $V$. Let $\Delta$ be the collection of disks guaranteed by Theorem 4.1. Then each component of $V \mid \Delta$ is a ball.

Proof. First, suppose that $V$ is cut along just one disk $D$. It is wellknown that the result is one or two handlebodies. Here is an outline of the proof, suggested by Saul Schleimer. Let $V^{\prime}$ be one component of the resulting manifold. Then $\pi_{1}\left(V^{\prime}\right)$ injects into $\pi_{1}(V)$, because if a loop $\gamma \subset V^{\prime}$ bounds a disk $E \subset V$, one can do disk swaps with $D$ to find a disk that $\gamma$ bounds in $V^{\prime}$ also. Thus $\pi_{1}\left(V^{\prime}\right)$ is free (as a subgroup of the free group $\left.\pi_{1}(V)\right)$, and so $V^{\prime}$ is a handlebody. Applying this argument repeatedly, we see that every component of $V \mid \Delta$ is a handlebody.

Let $X$ be one component of $V \mid \Delta . \partial X$ consists of a subset of $\partial V$ along with some number of distinguished disks $E_{1}, \ldots, E_{k}$ that come 
from removing $N(S)$. We already know that $X$ is a handlebody, and we prove that $X$ is a ball by showing that it contains no essential disks.

Suppose that $D \subset X$ is a disk with $\gamma=\partial D \subset \partial X$. Clearly, $D$ can be isotoped so that $\gamma$ is disjoint from the distinguished disks $E_{j}$. Thus $\gamma$ is a simple closed curve in $\partial V$ that bounds a disk $D \subset V$. By Theorem 4.1. some loop freely homotopic to $\gamma$ is generated by conjugates of the $\partial D_{i}$ in $\pi_{1}(\partial V)$. Passing to homology, we see that the class $[\gamma]$ is a linear combination of the $\left[\partial D_{i}\right]$ in $H_{1}(\partial V)$.

Let us express $\partial V$ as a union of open subsets $A$ and $B$, where $B=$ $\partial V \backslash \partial X$ and $A$ is an open regular neighborhood of $\partial X$ in $\partial V$. Thus $A \cap B$ is a disjoint union of open regular neighborhoods of the $\partial E_{j} \cdot \gamma$ lies in $A$ but is homologous to some cycle $c$ in $B$, because

$$
[\gamma]=\sum_{i=1}^{n} a_{i}\left[\partial D_{i}\right] \in H_{1}(\partial V) \text {, and } \partial D_{i} \subset B \text { for all } i \text {. }
$$

Now, the Mayer-Vietoris sequence gives us

$$
\ldots \rightarrow H_{1}(A \cap B) \stackrel{i_{*}}{\longrightarrow} H_{1}(A) \oplus H_{1}(B) \stackrel{j_{*}}{\longrightarrow} H_{1}(\partial V) \rightarrow \ldots
$$

induced by the chain maps $i(x)=(x, x)$ and $j(x, y)=x-y$. Since $[\gamma]-[c]=0 \in H_{1}(\partial V),([\gamma],[c]) \in \operatorname{ker}\left(j_{*}\right)$. But since the sequence is exact, $([\gamma],[c]) \in \operatorname{Im}\left(i_{*}\right)$. Thus $\gamma$ is homologous in $H_{1}(A)$ to a cycle that lies in $A \cap B$. But $H_{1}(A \cap B)$ is generated by the $\left[\partial E_{j}\right]$, so $\gamma$ must be homologically trivial in $\partial X$.

Since $X$ is a handlebody without any homologically essential disks, it must be a ball.

The following application of Meeks and Yau's theorem to a form of the Smith Conjecture for handlebodies was suggested by Ian Agol and Saul Schleimer.

Theorem 4.3. Let $V$ be a handlebody, and let $g: V \rightarrow V$ be an orientation-preserving, periodic diffeomorphism. Then

(1) The fixed-point set of $g$ is either empty or boundary-parallel.

(2) $W=V /(g)$ is also a handlebody, in which the image of the fixed-point set is again empty or boundary-parallel.

Proof. Let $\Delta$ be the collection of disks in $V$ given by Theorem 4.1 . By Lemma 4.2, $V \mid \Delta$ is a disjoint union of balls. The fixed-point set $a$ may be empty, or it may have one or more components. If $a$ is nonempty, we will prove that it is boundary-parallel by first considering its intersection with the individual balls and then seeing how the pieces join up.

For each component $B$ of $V \mid \Delta, \partial B$ consists of a subset of $\partial V$, together with some number of distinguished disks $E_{1}, \ldots, E_{k}$ that come 


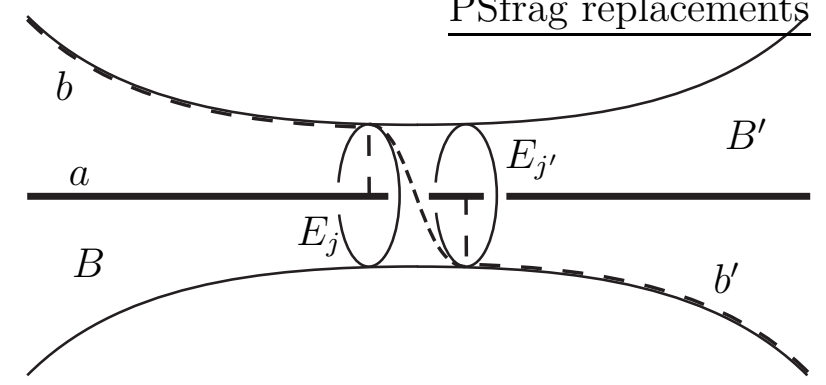

Figure 3. When the components of $V \mid \Delta$ are glued together along $\Delta$, the fixed-point locus $a$ remains isotopic to the boundary.

from removing $N(S)$. Since $V \mid \Delta$ is $g$-invariant, $g$ maps $B$ either onto itself or onto another ball. Thus if $B \cap a$ is non-empty, $g$ must send $B$ to itself.

Double $B$ along its boundary to get $S^{3}$. If $g$ maps $B$ to itself, its action will extend to an orientation-preserving, periodic, smooth map $h: S^{3} \rightarrow S^{3}$. By the solution to the Smith Conjecture [6], the fixedpoint set of $h$ is a single unknotted circle. Thus the double of $B \cap a$ is the unknot, making $B \cap a$ a single, boundary-parallel arc. We can choose the isotopic arc $b \subset \partial B$ so that it intersects the $E_{j}$ in a minimal way: if an endpoint of $B \cap a$ lies in some $E_{j}$, then $b \cap E_{j}$ is a radius of that disk. Otherwise, $b$ is disjoint from the distinguished disks.

To prove that $a$ is boundary-parallel in all of $V$, it remains to show how the isotopies in the individual balls extend across the disks in $\Delta$. If some $D_{i} \in \Delta$ is disjoint from $a$, it presents no problem. If $D_{i}$ does intersect $a$, it is preserved by $g$, and the intersection must be a point or an arc. If $D_{i}$ intersects $a$ in an arc, then that component of $a$ is already boundary-parallel through $D_{i}$. If $D_{i}$ intersects $a$ transversely, in a point, then the corresponding pair of disks $E_{j} \subset \partial B$ and $E_{j^{\prime}} \subset \partial B^{\prime}$ will each intersect it in a point too. In this case, the isotopies of $a \cap B$ and $a \cap B^{\prime}$ to $\partial V$ can be joined over $N\left(D_{i}\right)$, as in Figure 3. Thus any non-empty fixed-point locus $a$ must be boundary-parallel.

To prove part (2) of the theorem, consider the quotient of $V \mid \Delta$ under the action of $g$. This quotient is still a disjoint union of balls, which are glued along disks on their boundaries to reconstruct $W$. Thus $W$ can be viewed as a thickened graph, whose vertices are in the disjoint balls and whose edges correspond to gluings. As a thickened graph, $W$ must be a handlebody, and the image of $a$ under the quotient map is boundary-parallel by the same argument as above. 


\section{Proof of Theorem 1.1}

Lemma 3.1 proves the "if" direction of the theorem. To prove the "only if" direction, suppose that a strong involution $\psi$ of $K$ fixes its unknotting tunnel $\tau$ pointwise. By the Smith Conjecture solution, the fixed-point locus of $\psi$ is an unknotted circle. $\tau$ already lies on this axis; call the remaining arc of the axis $a$.

Notation. Let $\pi: S^{3} \rightarrow S^{3}$ be the quotient map induced by the action of $\psi$. Label $\hat{K}=\pi(K), \hat{\tau}=\pi(\tau)$, and $\hat{a}=\pi(a)$. Then $\pi$ is a branched covering map of $S^{3}$ by $S^{3}$, branched along the unknot $\hat{\tau} \cup \hat{a}$.

Recall the genus-2 Heegaard splitting of $S^{3}$ by $V_{1}=\overline{N(K \cup \tau)}$ and $V_{2}=E(K \cup \tau)$. $\psi$ acts as an involution on each $V_{i}$; let $W_{i}=\pi\left(V_{i}\right)$ be the quotients. Since $W_{1}$ is a closed regular neighborhood of the knot $\hat{K} \cup \hat{\tau}$, it is a solid torus. By Theorem 4.3, $W_{2}$ is a handlebody, and since $T=\partial W_{1}=\partial W_{2}$ is a torus, $W_{2}$ is itself a solid torus. The result now follows in two steps.

Claim 5.1. $\hat{K} \cup \hat{a}$ is a two-bridge knot with splitting sphere $\partial N(\hat{\tau})$.

Proof. The involution $\psi$ acts on each $V_{i}$ separately, and $a$ is the fixedpoint set of $\psi$ in $V_{2}$. By Theorem 4.3, it follows that $\hat{a}$ is boundaryparallel in $W_{2}$. Thus $W_{2} \backslash N(\hat{a})=E(\hat{K} \cup \hat{\tau} \cup \hat{a})$ is a genus-2 handlebody, and $\hat{\tau}$ is an unknotting tunnel for $\hat{K} \cup \hat{a}$. Furthermore, $\hat{\tau} \cup \hat{a}$ is the unknot by the solution to the Smith Conjecture, and $\hat{K} \cup \hat{\tau}$ is the unknot because $W_{2}=E(\hat{K} \cup \hat{\tau})$ is a solid torus. Thus Corollary 3.3 tells us that $\partial N(\hat{\tau})$ splits $\hat{K} \cup \hat{a}$ into rational tangles.

Claim 5.2. $K$ is a two-bridge knot, and $\tau$ is its upper or lower tunnel.

Proof. Claim 5.1 tells us that $\partial N(\hat{\tau})$ is a splitting sphere for $\hat{K} \cup \hat{a}$. In particular, $\hat{K} \cap E(\hat{\tau})$ is isotopic to $\partial N(\hat{\tau})$ via a disk $\hat{D}$ that is disjoint from $\hat{a}$. Since $\hat{D}$ is disjoint from the branch locus of $\pi$, it lifts to two disjoint disks, $D_{1}$ and $D_{2}$, that realize isotopies of $K_{1}$ and $K_{2}$, respectively, to $\partial N(\tau)$. Therefore, $(E(\tau), K \cap E(\tau))$ is a rational tangle and $K$ is a 2-bridge knot.

It follows that $\tau$ is an upper or lower tunnel for $K$, by the same argument as in Corollary 3.3 .

\section{REFERENCES}

[1] C. Adams. Unknotting tunnels in hyperbolic 3-manifolds. Math. Ann. 302 (1995), 177-195.

[2] S. Bleiler and Y. Moriah. Heegaard splittings and branched coverings of $B^{3}$. Math. Ann. 281 (1988), 531-543. 
[3] G. Burde and H. Zieschang. Knots. Berlin New York: de Gruyter, 1985.

[4] T. Kobayashi. Classification of unknotting tunnels for 2-bridge knots. In "Proceedings of the 1998 Kirbyfest", Geometry and Topology Monographs 2 (1999), 259-290.

[5] W. Meeks and S.-T. Yau. The equivariant Dehn's lemma and loop theorem. Comment. Math. Helv. 56 (1981), 225-239.

[6] J. Morgan and H. Bass, eds. The Smith Conjecture. New York: Academic Press, 1984.

[7] K. Morimoto and M. Sakuma. On unknotting tunnels for knots. Math. Ann. 289 (1991), 143-167.

[8] M. Scharlemann and A. Thompson. Detecting unknotted graphs in 3-space. J. Diff. Geom. 34 (1991), 539-560.

[9] H. Schubert. Knoten mit zwei Brücken. Math. Zeit. 66 (1956), 133-170.

Mathematics Department, Stanford University 